\title{
MATRIX TODA AND VOLTERRA LATTICES
}

\author{
AMÍLCAR BRANQUINHO ${ }^{\dagger}$, ANA FOULQUIÉ MORENO ${ }^{*}$, AND JUAN C. GARCÍA-ARDILA
}

AbSTRACT. We consider matrix Toda and Volterra lattice equations and their relation with matrix biorthogonal polynomials. From that relation, we give a method for constructing a new solution of these systems from another given one. An illustrative example is presented.

\section{INTRODUCTION}

The Toda lattice

$$
\left\{\begin{array}{l}
\dot{b}_{n}(t)=a_{n}(t)-a_{n+1}(t) \\
\dot{a}_{n+1}(t)=a_{n+1}(t)\left(b_{n}(t)-b_{n+1}(t)\right)
\end{array} \quad, \quad a_{0}=0, \quad n=0,1 \ldots,\right.
$$

when both $\left(a_{n}(t)\right)_{n \in \mathbb{N}}$ and $\left(b_{n}(t)\right)_{n \in \mathbb{N}}$ are real or complex functions has been well studied in the literature from different points of view (see [2, 3, 6, 11, 17, 19]). In particular, if we assume that $a_{n}(t) \neq 0, n>0$, and we define a sequence of polynomials $\left(p_{n}\right)_{n \in \mathbb{N}}$ recursively by

$$
p_{n+1}(x, t)=\left(x-b_{n}(t)\right) p_{n}(x, t)-a_{n}(t) p_{n-1}(x, t), \quad p_{-1}=0, \quad p_{0}=1,
$$

then using Favard's theorem [9], for each $t \in \mathbb{R}$. there exists a linear functional $u(t)$ such that the sequence of polynomials $\left(p_{n}\right)_{n \in \mathbb{N}}$ is orthogonal with respect to $u(t)$. This connection with orthogonal polynomials was used in [7, 11, 19] to give sufficient conditions for the construction of a solution of the above Toda lattice from another given one. Both solutions are linked by a Bäcklund transformation, given by

$$
\begin{array}{lll}
a_{n}=\gamma_{2 n-1} \gamma_{2 n}, & b_{n}=\gamma_{2 n}+\gamma_{2 n+1}+C, & n \in \mathbb{N}, \\
\widetilde{a}_{n}=\gamma_{2 n} \gamma_{2 n+1}, & \widetilde{b}_{n}=\gamma_{2 n+1}+\gamma_{2 n+2}+C, & n \in \mathbb{N},
\end{array}
$$

\footnotetext{
†Departamento de Matemática, Universidade de Coimbra, 3001-454 Coimbra, Portugal

* Departamento de Matemática, Universidade de Aveiro, 3810-193 Aveiro, Portugal

†Departamento de Ingeniería Civil: Hidráulica y Ordenación del Territorio E.T.S. De Ingeniería Civil, Universidad Politécnica de Madrid, Calle Alfonso XII, 3 y 5 Madrid, España.

E-mail addresses: ajplb@mat.uc.pt, foulquie@ua.pt, juancarlos.garciaa@upm.es.

2010 Mathematics Subject Classification. 42C05, 15A23, 30C10, 39B42, 34K08.

Key words and phrases. Matrix biorthogonal polynomials, matrix Toda lattice, matrix Volterra lattice, Symmetrized process, block Jacobi Matrices.
} 
where $\left(\gamma_{n}\right)_{n \in \mathbb{N}}$ is solution of the Volterra lattice

$$
\dot{\gamma}_{n+1}=\gamma_{n+1}\left(\gamma_{n}-\gamma_{n+2}\right), \quad n \in \mathbb{N} \text {. }
$$

Here we emphasize that the dot ". means differentiation with respect to $t \in \mathbb{R}$.

Both the Volterra and the new solution of the Toda lattices are strongly related with Darboux transformations of orthogonal polynomials (or equivalently, the LU and UL factorization of its Jacobi matrix associated [8]). In [5] the above analysis is generalized to high-order Toda and Volterra lattices.

The Darboux transformations has also been used by Spiridonov and Zhedanov to study the discrete-time Toda [20] and Volterra lattices [21] and their connection with AskeyWilson polynomials.

More recently, a matrix interpretation of high-order Toda lattices is given in [4] to consider the following semi-infinite system of differential equations

$$
\left\{\begin{array}{l}
\dot{a}_{n}=c_{n}-c_{n-2}, \\
\dot{b}_{n}=c_{n} a_{n+1}-c_{n-1} a_{n}+d_{n}-d_{n-2}, \\
\dot{c}_{n}=c_{n}\left(b_{n+1}-b_{n}\right)+d_{n} a_{n+2}-d_{n-1} a_{n}, \\
\dot{d}_{n}=d_{n}\left(b_{n+2}-b_{n}\right),
\end{array}\right.
$$

with initial conditions $a_{0}=b_{0}=c_{0}=d_{0}=c_{1}=0$, where $a_{n}, b_{n}, c_{n}$ and $d_{n}$ are complex functions depending on $t \in \mathbb{R}$. This system can be characterized in terms of matrix orthogonal polynomials satisfying the following three term recurrence relation

$$
x V_{m}(x)=A_{m+1} V_{m+1}+B_{n} V_{m}(x)+C_{m} V_{m-1}(x), \quad n \in \mathbb{N},
$$

where

$$
A_{m}=\left[\begin{array}{cc}
1 & 0 \\
a_{2 m+3} & 1
\end{array}\right], \quad B_{m}=\left[\begin{array}{ll}
b_{2 m+1} & a_{2 m+2} \\
c_{2 m+1} & b_{2 m+2}
\end{array}\right], \quad C_{m}=\left[\begin{array}{cc}
d_{2 m-1} & c_{2 m} \\
0 & d_{2 m}
\end{array}\right] .
$$

Notice that from the above differential system, (3) can be written in matrix notation as

$$
\left\{\begin{array}{l}
\dot{A}_{m}=A_{m} D_{m+1}-D_{m} A_{m} \\
\dot{B}_{m}=A_{m} C_{m+1}-C_{m} A_{m-1}+B_{m} D_{m}-D_{m} B_{m}, \quad n \in \mathbb{N}, \\
\dot{C}_{m}=B_{m} C_{m}-C_{m} B_{m-1}+C_{m} D_{m-1}-D_{m} C_{m}
\end{array}\right.
$$

where $D_{m}=\left[\begin{array}{cc}0 & 0 \\ c_{2 m+1} & 0\end{array}\right]$. With this new interpretation, the authors find (under some conditions) a representation of the vector of linear functional associated with the polynomials $\left(V_{n}\right)_{n \in \mathbb{N}}$ and show that the orthogonality governs the high-order Toda lattice.

In a more general framework, the non-Abelian Toda and Volterra lattices and the Bäcklund relation between them have been well studied [12, 13, 15, 16, 18], In particular, if we assume that $a_{n}$ and $b_{n}$ are matrices, then a solution of the Toda lattice can be expressed in term of quasideterminants [14] (the definition of quasideterminants can be found in [10]). 
In this paper we deal with similar Toda and Volterra lattices as in (1) and (2), but now with $N \times N$ matrix complex functions, $a_{n}, b_{n}$ and $\gamma_{n}$. In this case we need to be careful because the matrix product is no more commutative. Here, we also find a close relation between the matrix Toda lattice and the matrix biorthogonal polynomials associated to a matrix sesquilinear form, which allows us to find a new solution from a given one using the symmetrization process ( $c f$. Section 3). We also find a close relation between the matrix Toda and Volterra lattices.

This work is organized as follows: In Section 2, we present the basic theory about matrix biorthogonal polynomials and a Favard's matrix theorem. Following the ideas given in [9] we expose the symmetrization process for matrix sesquilinear forms associated to a matrix of the linear functionals, this process involves the block LU factorization (see [8] in the scalar case) and the matrix Christoffel transformation [1]. In Section 4, we study a matrix Toda system and use this symmetrization process (the analogous to the Bäcklund transformation) to construct a new solution from another given one. Both solutions are liked to each other by a matrix Volterra lattice. We also give a very instructive example which motivates the study in Section 5 of the matrix Volterra lattice (or equivalently matrix 2-Toda lattice), where we give characterizations of the solution of a Volterra lattice, its corresponding matrix of linear functionals associated to the block Jacobi matrix and its sequence of matrix biorthogonal polynomials.

\section{MATRIX BIORTHOGONAL POLYNOMIALS}

First of all we will fix some notation. Let $\mathbb{C}$ be the set of complex numbers, and denote by $\mathbb{C}^{N \times N}$ the linear space of $N \times N$ matrices with complex entries. For an arbitrary finite or semi-infinite matrix $A$, the matrix $A^{\dagger}$ is its transpose conjugate. We will denote by I and 0 the identity and zero $N \times N$ matrices, respectively.

A sesquilinear form on the bimodule of matrix polynomials $\mathbb{C}^{N \times N}[x]$, with real variable, is a map

$$
\langle\cdot, \cdot\rangle: \mathbb{C}^{N \times N}[x] \times \mathbb{C}^{N \times N}[x] \rightarrow \mathbb{C}^{N \times N},
$$

such that for any triple $P, Q, R \in \mathbb{C}^{N \times N}[x]$ and for all $A, B \in \mathbb{C}^{N \times N}$ :

1. $\langle A P(x)+B Q(x), R(x)\rangle=A\langle P(x), R(x)\rangle+B\langle Q(x), R(x)\rangle$;

2. $\langle P(x), A Q(x)+B R(x)\rangle=\langle P(x), Q(x)\rangle A^{\dagger}+\langle P(x), R(x)\rangle B^{\dagger}$.

If $\langle P(x), Q(x)\rangle=\langle Q(x), P(x)\rangle^{\dagger}$, then $\langle\cdot, \cdot\rangle$ is called a symmetric sesquilinear form.

Given a matrix of linear functionals, i.e.

$$
u=\left[\begin{array}{ccc}
u_{0,0} & \cdots & u_{0, N-1} \\
\vdots & \ddots & \vdots \\
u_{N-1,0} & \cdots & u_{N-1, N-1}
\end{array}\right]
$$


where $u_{i, j}$ belongs to the dual space of $\mathbb{C}[x]$, we define its associated sesquilinear form $\langle P, Q\rangle_{u}$ as follows

$$
\left(\langle P, Q\rangle_{u}\right)_{i, j}:=\sum_{k, l=0}^{N-1}\left[u_{k, l}, P_{i, k}(x) \bar{Q}_{j, l}(x)\right], \quad i, j=0,1 \cdots, N-1 .
$$

Here, $\left[u_{i, j}, p(x)\right]$ is the application of the linear functional $u_{i, j}$ to the scalar polynomial $p(x)$.

An important property of the sesquilinear form defined in terms of a matrix of linear functionals is that $\langle x P(x), Q(x)\rangle_{u}=\langle P(x), x Q(x)\rangle_{u}$. Hereinafter we only work with matrix sesquilinear forms satisfying the above property and unless otherwise stated we will assume that $\langle\mathrm{I}, \mathrm{I}\rangle_{u}=\mathrm{I}$.

Definition 1. The n-th moment of a sesquilinear form associated with a matrix of linear functionals is defined as

$$
u_{n}:=\left[\begin{array}{ccc}
\left\langle u_{0,0}, x^{n}\right\rangle & \ldots & \left\langle u_{0, N-1}, x^{n}\right\rangle \\
\vdots & \ddots & \vdots \\
\left\langle u_{N-1,0}, x^{n}\right\rangle & \ldots & \left\langle u_{N-1, N-1}, x^{n}\right\rangle
\end{array}\right], \quad n \in \mathbb{N} .
$$

We also define the block moment matrix $M$ and its $n$-th truncation as

$$
M:=\left[\begin{array}{ccc}
u_{0} & u_{1} & \cdots \\
u_{1} & u_{2} & \cdots \\
\vdots & \vdots & \ddots
\end{array}\right] . \quad M_{n}=\left[\begin{array}{ccc}
u_{0} & \cdots & u_{n-1} \\
\vdots & \cdots & \vdots \\
u_{n-1} & \cdots & u_{2 n-2}
\end{array}\right], \quad n \in \mathbb{N},
$$

Let $\chi(x):=\left[\begin{array}{llll}\mathrm{I} & \mathrm{I} x & \mathrm{I} x^{2} & \ldots\end{array}\right]^{\dagger}$. Observe that with this notation, the block moment matrix can be expressed as $M=\langle\chi(x), \chi(x)\rangle_{u}$.

Proposition 1. The block moment matrix $M$ satisfies

$$
M \Lambda^{\dagger}=\Lambda M \quad \text { where, } \Lambda=:\left[\begin{array}{cccc}
\mathbf{0} & \mathrm{I} & \mathbf{0} & \\
\mathbf{0} & \mathbf{0} & \mathrm{I} & \ddots \\
& \ddots & \ddots & \ddots
\end{array}\right] .
$$

Proof. The proof follows from the identity $\Lambda \chi(x)=x \chi(x)$.

Definition 2. A sesquilinear form $\langle\cdot, \cdot\rangle_{u}$ is said to be quasi-definite if all block leading sub-matrices of the corresponding block moment matrix are nonsingular.

Proposition 2 (cf. [1]). If the sesquilinear form $\langle\cdot, \cdot\rangle_{u}$ is quasi-definite, then its block moment matrix $M$ has an unique Gauss-Borel factorization,

$$
M=S_{1}^{-1} H\left(S_{2}\right)^{-\dagger} \text {, }
$$

where $S_{1}, S_{2}$ are lower unitriangular block matrices and $H$ is a block diagonal matrix. Moreover, if $M=M^{\dagger}$, then $S_{1}=S_{2}$. 
Definition 3. Given a quasi-definite sesquilinear form $\langle\cdot, \cdot\rangle_{u}$ such that the associated block moment matrix has a Gauss-Borel factorization as in (5), then we define the corresponding first and second families of matrix biorthogonal polynomials as follows

$$
P^{[1]}(x)=\left[\begin{array}{c}
P_{0}^{[1]}(x) \\
P_{1}^{[1]}(x) \\
\vdots
\end{array}\right]:=S_{1} \chi(x), \quad P^{[2]}(x)=\left[\begin{array}{c}
P_{0}^{[2]}(x) \\
P_{1}^{[2]}(x) \\
\vdots
\end{array}\right]:=S_{2} \chi(x) .
$$

Proposition 3 (Biorthogonality). The first and second families of monic matrix polynomials $\left(P_{n}^{[1]}\right)_{n \in \mathbb{N}}$ and $\left(P_{n}^{[2]}\right)_{n \in \mathbb{N}}$ are biorthogonal, i.e.,

$$
\left\langle P_{n}^{[1]}(x), P_{m}^{[2]}(x)\right\rangle_{u}=\delta_{n, m} H_{n}, \quad n, m \in \mathbb{N},
$$

where $H_{n}$ is the $(n, n)$-block element of the block semi-infinite matrix $H$ obtained in the Gauss-Borel factorization (5). These biorthogonal relations yields,

$$
\begin{array}{lll}
\left\langle P_{n}^{[1]}(x), x^{m} \mathrm{I}\right\rangle_{u}=\mathbf{0}, & \left\langle x^{m} \mathrm{I}, P_{n}^{[2]}(x)\right\rangle_{u}=\mathbf{0}, & m=0, \ldots, n-1, \\
\left\langle P_{n}^{[1]}(x), x^{n} \mathrm{I}\right\rangle_{u}=H_{n}, & \left\langle x^{n} \mathrm{I}, P_{n}^{[2]}(x)\right\rangle_{u}=H_{n}, & n \in \mathbb{N} .
\end{array}
$$

Definition 4. The matrices

$$
J_{1}:=S_{1} \Lambda S_{1}^{-1}, \quad J_{2}:=S_{2} \Lambda S_{2}^{-1},
$$

are said to be the Jacobi matrices associated with the moment matrix $M$.

Proposition 4. The two Jacobi matrices in (6) are related by

$$
H^{-1} J_{1}=J_{2}^{\dagger} H^{-1},
$$

being block tridiagonal. Moreover, we have that

$$
J_{1} P^{[1]}(x)=x P^{[1]}(x), \quad J_{2} P^{[2]}(x)=x P^{[2]}(x) .
$$

Proof. The relation between the above two Jacobi matrices follows from the Gauss-Borel factorization and (4). The relation (7) follows from the definitions of the Jacobi matrices (6).

Observe that a consequence of the above proposition is that $J_{1}$ and $J_{2}$ have a three diagonal block shape with the block I on the superdiagonal,

$$
J_{1}=\left[\begin{array}{cccc}
b_{0}^{[1]} & \mathrm{I} & & \\
a_{1}^{[1]} & b_{1}^{[1]} & \mathrm{I} & \\
& \ddots & \ddots & \ddots
\end{array}\right], \quad J_{2}=\left[\begin{array}{cccc}
b_{0}^{[2]} & \mathrm{I} & & \\
a_{1}^{[2]} & b_{1}^{[2]} & \mathrm{I} & \\
& \ddots & \ddots & \ddots
\end{array}\right] .
$$


The equations in (7) means that $\left(P_{n}^{[1]}\right)_{n \in \mathbb{N}}$ and $\left(P_{n}^{[2]}\right)_{n \in \mathbb{N}}$, respectively satisfies a three term recurrence relation

$$
\begin{array}{lll}
x P_{n}^{[1]}(x)=P_{n+1}^{[1]}(x)+b_{n}^{[1]} P_{n}^{[1]}(x)+a_{n}^{[1]} P_{n-1}^{[1]}(x), & P_{0}^{[1]}(x)=\mathrm{I}, & P_{-1}^{[1]}(x)=\mathbf{0}, \\
x P_{n}^{[2]}(x)=P_{n+1}^{[2]}(x)+b_{n}^{[2]} P_{n}^{[2]}(x)+a_{n}^{[2]} P_{n-1}^{[2]}(x), & P_{0}^{[2]}(x)=\mathrm{I}, & P_{-1}^{[2]}(x)=\mathbf{0},
\end{array}
$$

where for $i=1,2, a_{n}^{[i]}$, and $b_{n}^{[i]}$ are $N \times N$ matrices. Moreover, $a_{n}^{[1]}=H_{n} H_{n-1}^{-1}$.

Proposition 5. Given a matrix of linear functionals $u$ and its sequence of moments $\left(u_{n}\right)_{n \in \mathbb{N}}$. Then $u_{n}=\left(J_{1}^{n}\right)_{0,0}, n \in \mathbb{N}$.

Proof. We know from (7) that $J_{1}^{n} P^{[1]}(x)=x^{n} P^{[1]}(x), n \in \mathbb{N}$, and the first line gives us

$$
x^{n} P_{0}^{[1]}(x)=\sum_{k=0}^{n}\left(J_{1}^{n}\right)_{0, k} P_{k}^{[1]}(x) ;
$$

applying the sesquilinear form and making use of the biorthogonality condition we get the desired result.

Definition 5 (cf. [10]). Let $A \in \mathbb{C}^{m \times m}, B \in \mathbb{C}^{m \times N}, C \in \mathbb{C}^{N \times m}$ and $D \in \mathbb{C}^{N \times N}$, with $A$ a nonsingular matrix. If we take the block matrix $\left[\begin{array}{ll}A & B \\ C & D\end{array}\right]$, then

$$
\Theta_{*}\left[\begin{array}{cc}
A & B \\
C & D
\end{array}\right]=D-C A^{-1} B
$$

is said to be the last quasi-determinant of the matrix $\left[\begin{array}{cc}A & B \\ C & D\end{array}\right]$.

Remark 1. The sequences of matrix polynomials $\left(P_{n}^{[1]}\right)_{n \in \mathbb{N}}$ and $\left(H_{n}\right)_{n \in \mathbb{N}}$ can be written in term of the moments $\left(u_{n}\right)_{n \in \mathbb{N}}$ as follow (see [1])

$$
P_{n}^{[1]}(x)=: x^{n} I_{p}-\left[\begin{array}{lll}
u_{n} & \cdots & u_{2 n-1}
\end{array}\right] M_{n}^{-1}\left[\begin{array}{c}
I_{p} \\
x I_{p} \\
\vdots \\
x^{n-1} I_{p}
\end{array}\right]
$$

and

$$
H_{n}=\Theta_{*}\left[\begin{array}{c|c} 
& u_{n} \\
M_{n} & \vdots \\
& u_{2 n-1} \\
\hline u_{n} \cdots u_{2 n-1} & u_{2 n}
\end{array}\right]
$$


Using this fact and the thee term recurrence relation, we have

$$
a_{n}^{[1]}=H_{n} H_{n-1}^{-1}, \quad b_{n}^{[1]}=D_{n} H_{n}, \quad \text { where } \quad D_{n}=\Theta_{*}\left[\begin{array}{c|c}
u_{n+1} & \vdots \\
& u_{2 n} \\
\hline u_{n} \cdots u_{2 n-1} & u_{2 n+1}
\end{array}\right] .
$$

There exist similar formulas for $\left(P_{n}^{[2]}\right)_{n \in \mathbb{N}}, a_{n}^{[2]}$ and $b_{n}^{[2]}$.

Definition 6. Let $u$ be a matrix of linear functionals and its sequence of moments $\left(u_{n}\right)_{n \in \mathbb{N}}$. If $r:=\sup \{|x|: x \in \operatorname{supp} u\})<\infty$ we consider the disks about infinity, $\mathbb{D}:=\{z \in \mathbb{C}:|z|>r\}$. Then, for $z \in \mathbb{D}$ we define the Stieltjes matrix function as

$$
F(z)=\left\langle\frac{1}{z-x} \mathrm{I}, \mathrm{I}\right\rangle_{u} .
$$

Observe that in this definition we have that the geometric series $\sum_{k=0}^{\infty}\left(\frac{x}{z}\right)^{k}$ is uniform convergent in any compact subset of $\mathbb{D}$. With this in mind, $F(z)$ can also be written as

$$
F(z)=\sum_{k=0}^{\infty} \frac{u_{k}}{z^{k+1}} .
$$

Proposition 6 (Matrix Favard's theorem). Let $\left(a_{n}\right)_{n \in \mathbb{N}}$ and $\left(b_{n}\right)_{n \in \mathbb{N}}$ be two sequences of matrices with $a_{n}$ nonsingular for every $n \in \mathbb{N}$. If we define the matrix polynomials $\left(P_{n}^{[1]}\right)_{n \in \mathbb{N}}$ by

$$
\begin{aligned}
& P_{n+1}^{[1]}(x)=\left(x \mathrm{I}-b_{n}\right) P_{n}^{[1]}(x)-a_{n} P_{n-1}^{[1]}(x), \quad n \geq 0, \\
& P_{0}(x)=\mathrm{I}, \quad P_{-1}(x)=\mathbf{0},
\end{aligned}
$$

then we can find a matrix of linear functionals $u$ such that its sesquilinear form satisfies

$$
\left\langle P_{n}^{[1]}(x), x^{m} I\right\rangle_{u}=\left(a_{n} \cdots a_{1}\right) \delta_{n, m},
$$

where $\delta_{n, m}$ is the Kronecker delta function.

Proof. To prove the above result we define the moments $\left(u_{n}\right)_{n \in \mathbb{N}}$ of the matrix of the linear functional $u$ inductively by

$$
u_{0}=\langle\mathrm{I}, \mathrm{I}\rangle_{u}=\mathrm{I}, \quad\left\langle P_{n}^{[1]}(x), \mathrm{I}\right\rangle_{u}=\mathbf{0}, \quad n=1,2 \ldots
$$

So, we can define $u_{1}$ using the fact that

$$
\mathbf{0}=\left\langle P_{1}^{[1]}(x), \mathrm{I}\right\rangle_{u}=\left\langle\left(x \mathrm{I}-b_{0}\right), \mathrm{I}\right\rangle=u_{1}-b_{0} u_{0} .
$$

In the same way $u_{2}$ can be defined by

$$
\mathbf{0}=\left\langle P_{2}^{[1]}(x), \mathrm{I}\right\rangle_{u}=\left\langle x^{2} \mathrm{I}-x b_{1}-x b_{0}-b_{1} b_{0}, \mathrm{I}\right\rangle_{u}=u_{2}-\left(b_{0}+b_{1}\right) u_{1}-b_{1} b_{0} u_{0} .
$$


Following this process we can find all the moments of $u$. From the above definition and the recurrence relation (9), we have

$$
\left\langle P_{n}^{[1]}(x), x^{m} \mathrm{I}\right\rangle_{u}=\mathbf{0}, \quad m<n, \quad \text { and } \quad H_{n}=\left\langle P_{n}^{[1]}(x), x^{n} \mathrm{I}\right\rangle_{u}=a_{n} \cdots a_{1} .
$$

As a last comment, it is important to point out that from the Proposition 4 , we can construct the second family of matrix biorthogonal polynomials with respect to $\langle\cdot, \cdot\rangle_{u}$, i.e. $\left(P_{n}^{[2]}\right)_{n \in \mathbb{N}}$.

\section{SYMMETRIZED SESQUILINEAR FORMS}

Let $\left(P_{n}^{[1]}\right)_{n \in \mathbb{N}}$ and $\left(P_{n}^{[2]}\right)_{n \in \mathbb{N}}$ be the sequences of matrix biorthogonal polynomials with respect to a quasi-definite sesquilinear form $\langle\cdot, \cdot\rangle_{u}$. We are interested in finding conditions such that the Jacobi matrices $J_{1}$ and $J_{2}$, have block LU factorization, i.e,

$$
J_{i}=L_{i} U_{i}=\left[\begin{array}{cccc}
\mathrm{I} & & & \\
\gamma_{2}^{[i]} & \mathrm{I} & & \\
& \gamma_{4}^{[i]} & \mathrm{I} & \\
& & \ddots & \ddots
\end{array}\right]\left[\begin{array}{cccc}
\gamma_{1}^{[i]} & \mathrm{I} & & \\
& \gamma_{3}^{[i]} & \mathrm{I} & \\
& & \ddots & \ddots
\end{array}\right], \quad i=1,2 .
$$

The above factorization will be our principal tool to construct a new solution of a Matrix Toda system (as in (17), Section 4 from another given one.

Lemma 1. Let $J_{i}, i=1,2$, be the Jacobi matrix associated to $\left(P_{n}^{[1]}\right)_{n \in \mathbb{N}}$. If $P_{n}^{[i]}(0)$ is a nonsingular matrix, then there exist block matrices $L_{i}$ and $U_{i}$ as in (10) such that $J_{i}=L_{i} U_{i}$. Moreover,

$$
\gamma_{2 n+1}^{[i]}=-P_{n+1}^{[i]}(0)\left(P_{n}^{[i]}(0)\right)^{-1}, \quad n \in \mathbb{N} .
$$

Proof. If the factorization exists, then necessarily

$$
b_{n}^{[i]}=\left(\gamma_{2 n+1}^{[i]}+\gamma_{2 n}^{[i]}\right), \quad n \geq 0, \quad a_{n}^{[i]}=\gamma_{2 n}^{[i]} \gamma_{2 n-1}^{[i]}, \quad n \geq 1,
$$

with $\gamma_{0}=\mathbf{0}$. Now, we are going to use the induction process to prove (11). Observe that for $n=0, b_{0}^{[i]}=-P_{1}^{[i]}(0)\left(P_{0}^{[i]}(0)\right)^{-1}=\gamma_{1}^{[i]}$. Suppose now that for $k \leq n, \gamma_{2 k-1}^{[i]}=$ $-P_{k}^{[i]}(0)\left(P_{k-1}^{[i]}(0)\right)^{-1}$. Using the three term recurrence relation (8)

$$
-P_{n+1}^{[i]}(0)=b_{n}^{[i]} P_{n}^{[i]}(0)+a_{n}^{[i]} P_{n-1}^{[i]}(0)
$$

then

$$
\begin{aligned}
-P_{n+1}^{[i]}(0)\left(P_{n}^{[i]}(0)\right)^{-1} & =b_{n}^{[i]}+a_{n}^{[i]} P_{n-1}^{[i]}(0)\left(P_{n}^{[i]}(0)\right)^{-1} \\
& =b_{n}^{[i]}+a_{n}^{[i]}\left[P_{n}^{[i]}(0)\left(P_{n-1}^{[i]}(0)\right)^{-1}\right]^{-1} \\
& =b_{n}^{[i]}-\gamma_{2 n}^{[i]} \gamma_{2 n-1}^{[i]}\left(\gamma_{2 n-1}^{[i]}\right)^{-1} \\
& =b_{n}^{[i]}-\gamma_{2 n}^{[i]}=\gamma_{2 n+1}^{[i]} .
\end{aligned}
$$


Thus, if $P_{n}^{[i]}(0)$, is a nonsingular matrix, then the block elements of the matrix $U_{i}$ are well defined. Defining the block elements of the matrix $L_{i}$ as $\gamma_{2 n}^{[i]}=: b_{n}^{[i]}-\gamma_{2 n+1}^{[i]}$ we obtain the result.

Define now $\widetilde{J_{i}}=U_{i} L_{i}, i=1,2$. It is not difficult to check that $\widetilde{J}_{i}$ is also a block tridiagonal matrix

$$
\widetilde{J}_{i}=\left[\begin{array}{ccccc}
\widetilde{b}_{0}^{[i]} & \mathrm{I} & & & \\
\widetilde{a}_{1}^{[i]} & \widetilde{b}_{1}^{[i]} & \mathrm{I} & & \\
& \widetilde{a}_{2}^{[i]} & \widetilde{b}_{2}^{[i]} & \mathrm{I} & \\
& & \ddots & \ddots & \ddots
\end{array}\right],
$$

thus the sequences $\left(\widetilde{P}_{n}^{[i]}\right)_{n \in \mathbb{N}}, i=1,2$, of matrix polynomials defined by the recurrence formula

$$
\begin{aligned}
x \widetilde{P}_{n}^{[i]}(x) & =\widetilde{P}_{n+1}^{[i]}(x)+\widetilde{b}_{n}^{[i]} \widetilde{P}_{n}^{[i]}(x)+\widetilde{a}_{n}^{[i]} \widetilde{P}_{n-1}^{[i]}(x), n \geq 0, \\
\widetilde{P}_{-1}^{[i]}(x) & =\mathbf{0}, \quad \widetilde{P}_{0}^{[i]}(x)=\mathrm{I},
\end{aligned}
$$

are also biorthogonal with respect to a matrix sesquilinear form, but what is the matrix of the functionals? The answer is given in the next result.

Proposition 7. Let $u$ be a matrix of linear functionals and $J_{i}, i=1,2$ its respectively block Jacobi matrices with block LU factorization as in (10), (or equivalently, for every $n \in \mathbb{N}, P_{n}^{[i]}(0)$ is a nonsingular matrix). Then the matrix polynomials $\left(\widetilde{P}_{n}^{[1]}\right)_{n \in \mathbb{N}}$ and $\left(\widetilde{P}_{n}^{[2]}\right)_{n \in \mathbb{N}}$ associated to $\widetilde{J}_{1}=U_{1} L_{1}$ and $\widetilde{J}_{2}=U_{2} L_{2}$ are the sequences of matrix biorthogonal polynomials with respect to the sesquilinear form generated by the matrix of linear functionals $x u$.

Proof. Define $Q^{[i]}=U_{i} P^{[i]}$ with $i=1,2$. The hypothesis implies that $Q_{n}^{[i]}(x)=P_{n+1}^{[i]}(x)+$ $\gamma_{2 n+1}^{[i]} P_{n}^{[i]}(x)$ (observe that $Q_{n}^{[i]}(x)$ is a polynomial of degree $n+1$ ). From the block LU factorization we also have that $x P^{[i]}=\left(L_{i} U_{i}\right) P^{[i]}=L_{i} Q^{[i]}$, thus

$$
x P_{n}^{[i]}(x)=Q_{n}^{[i]}(x)+\gamma_{2 n}^{[i]} Q_{n-1}^{[i]}(x), \quad n \in \mathbb{N},
$$

but this implies that $Q_{n}^{[i]}(0)=-\gamma_{2 n}^{[i]} Q_{n-1}^{[i]}(0)$ and taking into account that by definition $\gamma_{0}^{[i]}=\mathbf{0}$, then $Q_{n}^{[i]}(0)=\mathbf{0}$ for every $n=0,1, \ldots$ From here, $Q_{n}^{[i]}(x)$ can be written as $x \widetilde{P}_{n}^{[i]}(x)$ where $\widetilde{P}_{n}^{[i]}(x)$ is a matrix polynomial of degree $n$. As a consequence

$$
x \widetilde{P}_{n}^{[i]}(x)=P_{n+1}^{[i]}(x)+\gamma_{2 n+1}^{[i]} P_{n}^{[i]}(x) .
$$

with $\gamma_{2 n+1}^{[i]}=-P_{n+1}^{[i]}(0)\left(P_{n}^{[i]}(0)\right)^{-1}$. In [1] was shown that $\left(\widetilde{P}_{n}^{[1]}\right)_{n \in \mathbb{N}}$ and $\left(\widetilde{P}_{n}^{[2]}\right)_{n \in \mathbb{N}}$ are precisely the families of matrix biorthogonal polynomials associated to the sesquilinear form $\langle\cdot, \cdot\rangle_{x u}$. 
Definition 7 (cf. [1]). The matrix of linear functionals $x u$ is said to be the Christoffel transformation of $u$.

If now, we define the sequences of matrix polynomials $\left(S_{n}^{[1]}\right)_{n \in \mathbb{N}}$ and $\left(S_{n}^{[2]}\right)_{n \in \mathbb{N}}$ by

$$
S_{2 n}^{[i]}(x)=P_{n}^{[i]}\left(x^{2}\right), \quad S_{2 n+1}^{[i]}(x)=x \widetilde{P}_{n}^{[i]}\left(x^{2}\right), \quad i=1,2,
$$

it is not difficult to check that $\left(S_{n}^{[i]}\right)_{n \in \mathbb{N}}$ for $i=1,2$, satisfies the following three term recurrence relation

$$
x S_{n}^{[i]}(x)=S_{n+1}^{[i]}(x)+\gamma_{n}^{[i]} S_{n-1}^{[i]}(x), \quad n \in \mathbb{N},
$$

or equivalently, its corresponding block Jacobi matrix has the shape

$$
\Gamma_{1}^{[i]}=\left[\begin{array}{cccccc}
\mathbf{0} & \mathrm{I} & & & & \\
\gamma_{1}^{[i]} & \mathbf{0} & \mathrm{I} & & & \\
\mathbf{0} & \gamma_{2}^{[i]} & \mathbf{0} & \mathrm{I} & & \\
& \ddots & \ddots & \ddots & \ddots & \ddots
\end{array}\right], \quad i=1,2 .
$$

Let $\mathfrak{s}$ be the matrix of linear functionals for which the sequences $\left(S_{n}^{[1]}\right)_{n \in \mathbb{N}}$ and $\left(S_{n}^{[2]}\right)_{n \in \mathbb{N}}$ are biorthogonal and $\left(w_{n}\right)_{n \in \mathbb{N}}$ its corresponding sequence of matrix moments, then

$$
w_{2 n}=u_{n} \quad \text { and } \quad w_{2 n+1}=\mathbf{0}, \quad n \in \mathbb{N} .
$$

Observe also that from the block LU factorization, we have the following relations for $i=1,2$,

$$
\begin{aligned}
& b_{n}^{[i]}=\left(\gamma_{2 n+1}^{[i]}+\gamma_{2 n}^{[i]}\right), \quad n \geq 0, \quad \quad a_{n}^{[i]}=\gamma_{2 n}^{[i]} \gamma_{2 n-1}^{[i]}, \quad n \geq 1, \\
& \widetilde{b}_{n}^{[i]}=\left(\gamma_{2 n+2}^{[i]}+\gamma_{2 n+1}^{[i]}\right), \quad n \geq 0, \quad \widetilde{a}_{n}^{[i]}=\gamma_{2 n+1}^{[i]} \gamma_{2 n}^{[i]} \quad n \geq 1 \text {, }
\end{aligned}
$$

with the convention $\gamma_{0}^{[i]}=\mathbf{0}$. From here and Proposition 7

$$
\begin{array}{ll}
\gamma_{2 n+1}^{[i]}=-P_{n+1}^{[i]}(0)\left(P_{n}^{[i]}(0)\right)^{-1}, & \gamma_{2 n}^{[i]}=-a_{n}^{[i]} P_{n-1}^{[i]}(0)\left(P_{n}^{[i]}(0)\right)^{-1}, \\
\gamma_{2 n+1}^{[2]}=\left(H_{n}^{-1} \gamma_{2 n+1}^{[1]} H_{n}\right)^{\dagger}, & \gamma_{2 n}^{[2]}=\left(H_{n}^{-1} \gamma_{2 n}^{[1]} H_{n}\right)^{\dagger} .
\end{array}
$$

The above, give us the following representation for $\widetilde{a}_{n}^{[i]}$ and $\widetilde{b}_{n}^{[i]}$

$$
\begin{aligned}
& \widetilde{b}_{n}^{[i]}=P_{n+2}^{[i]}(0)\left(P_{n+1}^{[i]}(0)\right)^{-1}+b_{n+1}^{[i]}-P_{n+1}^{[i]}(0)\left(P_{n}^{[i]}(0)\right)^{-1}, \\
& \widetilde{a}_{n}^{[i]}=P_{n+1}^{[i]}(0)\left(P_{n}^{[i]}(0)\right)^{-1} a_{n}^{[i]} P_{n-1}^{[i]}(0)\left(P_{n}^{[i]}(0)\right)^{-1} .
\end{aligned}
$$




\section{Matrix Toda Lattice}

In the scalar case, if we have a moment functional $\mu(t): \mathbb{C}[x] \rightarrow \mathbb{C}$ depending of a parameter $t \in \mathbb{R}$, then it is clear that its moments also dependent of $t$ i.e $\left\langle\mu(t), x^{n}\right\rangle=\mu_{n}(t)$. We can define the derivative of a moment functional with respect to $t$ as follows,

$$
\left\langle\frac{d}{d t} \mu(t), p(x)\right\rangle=\lim _{h \rightarrow 0} \frac{\langle\mu(t+h), p(x)\rangle-\langle\mu(t), p(x)\rangle}{h}, \quad p(x) \in \mathbb{C}[x] .
$$

An important property that can be proven from the above definition is the following. Suppose that we have a polynomial depending of $t$, i.e $p(x, t)=\sum_{k=0}^{m} c_{k}(t) x^{k}$ then

$$
\frac{d}{d t}\langle\mu(t), p(x, t)\rangle=\left\langle\frac{d}{d t} \mu(t), p(x, t)\right\rangle+\left\langle\mu(t), \frac{d}{d t} p(x, t)\right\rangle .
$$

Extrapolating the above argument, we can define the derivative of a sesquilinear form associated to a matrix of linear functionals that depends on a parameter $t$ as follows

$$
\frac{d}{d t}\langle P(x), Q(x)\rangle_{u(t)}
$$

$$
=\left\langle\frac{d}{d t} P(x), Q(x)\right\rangle_{u(t)}+\langle P(x), Q(x)\rangle_{\frac{d}{d t} u(t)}+\left\langle P(x), \frac{d}{d t} Q(x)\right\rangle_{u(t)} .
$$

Consider now the following semi-infinite system of matrix differential equations

$$
\left\{\begin{array}{l}
\dot{b}_{n}(t)=a_{n}(t)-a_{n+1}(t), \\
\dot{a}_{n+1}(t)=a_{n+1}(t) b_{n}(t)-b_{n+1}(t) a_{n+1}(t)
\end{array} \quad, \quad a_{0}(t)=\mathbf{0}, \quad n \in \mathbb{N} .\right.
$$

To give an interpretation of the above system, we start by considering the following sequence of matrix orthogonal polynomials $\left(P_{n}^{[1]}\right)_{n \in \mathbb{N}}$ satisfying the three term recurrence relation

$$
\begin{aligned}
& x P_{n}^{[1]}(x, t)=P_{n+1}^{[1]}(x, t)+b_{n}(t) P_{n}^{[1]}(x, t)+a_{n}(t) P_{n-1}^{[1]}(x, t) \quad n \geq 0, \\
& P_{-1}^{[1]}(x, t)=\mathbf{0}, \quad P_{0}^{[1]}(x, t)=\mathrm{I} .
\end{aligned}
$$

From Proposition 6, we know that there exists a sesquilinear form $\langle\cdot, \cdot\rangle_{u}$ (depending on $t$ ) such that the sequence $\left(P_{n}^{[1]}\right)_{n \in \mathbb{N}}$ is the first family of matrix biorthogonal polynomials, as well as that the sequences $\left(a_{n}(t)\right)_{n \in \mathbb{N}}$ and $\left(b_{n}(t)\right)_{n \in \mathbb{N}}$ can be written in term of the quasideterminants ( $c f$. Remark 1).

In this Section we are interested in showing the relationship that there exists between the sequence of matrix polynomials $\left(P_{n}^{[1]}\right)_{n \in \mathbb{N}}$ (as well as, its respective matrix of the linear functionals associated) and the Toda matrix system (17). 
If we assume that $J_{1}$ is as in (8), we have that the system (17) can be described in terms of a Lax pair $\left(J_{1}, J_{1-}\right)$, i.e.

$$
\dot{J}_{1}(t)=J_{1-} J_{1}-J_{1} J_{1-},
$$

where $J_{1-}$ is the following block matrix

$$
J_{1-}=\left[\begin{array}{cccc}
\mathbf{0} & \mathbf{0} & \mathbf{0} & \\
a_{1}(t) & \mathbf{0} & \mathbf{0} & \\
\mathbf{0} & a_{2}(t) & \mathbf{0} & \\
& \ddots & \ddots & \ddots
\end{array}\right] .
$$

Lemma 2. Let $J_{1}$ be a solution of a Toda lattice system as in (17). Then, the following relation holds,

$$
\dot{H}_{n}=-b_{n} H_{n}+H_{n} b_{0}, \quad n \in \mathbb{N} .
$$

Proof. Taking into account that $a_{n}(t)=H_{n} H_{n-1}^{-1}$ then from 17 we get

$$
\dot{H}_{n} H_{n-1}^{-1}-H_{n} H_{n-1}^{-1} \dot{H}_{n-1} H_{n-1}^{-1}=H_{n} H_{n-1}^{-1} b_{n-1}-b_{n} H_{n} H_{n-1}^{-1} \text {. }
$$

After some manipulations, we arrive to

$$
H_{n}^{-1} \dot{H}_{n}-H_{n-1}^{-1} \dot{H}_{n-1}=H_{n-1}^{-1} b_{n-1} H_{n-1}-H_{n}^{-1} b_{n} H_{n} .
$$

Thus, for every $n \in \mathbb{N}$ we see that

$$
H_{n}^{-1}\left(\dot{H}_{n}+b_{n} H_{n}\right)=H_{0}^{-1}\left(\dot{H}_{0}+b_{0} H_{0}\right),
$$

and remembering that $H_{0}=\mathrm{I}$, we complete the proof.

Proposition 8. If $J_{1}$ is a solution of a Toda lattice as in (17), then $J_{2}$ is a solution of the semi-infinite matrix differential system

$$
\left\{\begin{array}{l}
\dot{b}_{n}^{[2]}=\left(a_{n}^{[2]}-a_{n+1}^{[2]}\right)+\left(b_{0}^{[2]} b_{n}^{[2]}-b_{n}^{[2]} b_{0}^{[2]}\right) \\
\dot{a}_{n+1}^{[2]}=a_{n+1}^{[2]} b_{n}^{[2]}-b_{n+1}^{[2]} a_{n+1}^{[2]}+\left(b_{0}^{[2]} a_{n+1}^{[2]}-a_{n+1}^{[2]} b_{0}^{[2]}\right)
\end{array}, \quad a_{0}^{[2]}=\mathbf{0}, \quad n \in \mathbb{N} .\right.
$$

Proof. From Proposition 4 we have that

$$
\begin{aligned}
\dot{J}_{2}^{\dagger} & =-\dot{H}^{-1} J_{1} H+H^{-1} \dot{J}_{1} H+H^{-1} J_{1} \dot{H} \\
& =-H^{-1} \dot{H}\left(H^{-1} J_{1} H\right)+H^{-1}\left(J_{1-} J_{1}-J_{1} J_{1-}\right) H+H^{-1} J_{1} \dot{H} \\
& =\left(H^{-1} J_{1-} H-H^{-1} \dot{H}\right) J_{2}^{\dagger}-J_{2}^{\dagger}\left(H^{-1} J_{1-} H-H^{-1} \dot{H}\right) .
\end{aligned}
$$

On the other hand, from Lemma 2 we get

$$
H^{-1} J_{1-} H-H^{-1} \dot{H}=\left[\begin{array}{ccccc}
\mathbf{0} & & & & \\
\mathrm{I} & \left(b_{1}^{[2]}-b_{0}^{[2]}\right)^{\dagger} & & \\
\mathbf{0} & \mathrm{I} & \left(b_{2}^{[2]}-b_{0}^{[2]}\right)^{\dagger} & \\
& \ddots & \ddots & \ddots
\end{array}\right] .
$$


Thus, replacing the above in (21) we obtain

$$
\left(\dot{J}_{2}^{\dagger}\right)_{n, m}=\left\{\begin{array}{lll}
\left(a_{n}^{[2] \dagger}-a_{n+1}^{[2] \dagger}\right)+\left(b_{n}^{[2] \dagger} b_{0}^{[2] \dagger}-b_{0}^{[2] \dagger} b_{n}^{[2] \dagger}\right), & \text { if } & m=n, \\
b_{n}^{[2] \dagger} a_{n+1}^{[2] \dagger}-a_{n+1}^{[2] \dagger} b_{n+1}^{[2] \dagger}+\left(a_{n+1}^{[2] \dagger} b_{0}^{[2 \dagger]}-b_{0}^{[2] \dagger} a_{n+1}^{[2] \dagger}\right), & \text { if } & m=n+1,
\end{array}\right.
$$

and the result follows.

From Proposition 8 we note that $J_{2}$ is not a solution of the matrix Toda lattice. However it is true if we apply a transformation as we will see in the next result.

Proposition 9. Let $J_{1}$ be a solution of a Toda lattice system as in (17). If for every $H_{n}$, we can find two matrix functions $V_{n}$ and $W_{n}$ satisfying

$$
\dot{V}_{n}=-b_{n} V_{n}, \quad \dot{W}_{n}=-b_{0} W_{n},
$$

and such that $H_{n}=V_{n} W_{n}^{-1}$, then

$$
\begin{cases}\frac{d}{d t}\left(W_{0}^{\dagger} b_{n}^{[2]} W_{0}^{-\dagger}\right)=W_{0}^{\dagger} a_{n}^{[2]} W_{0}^{-\dagger}-W_{0}^{\dagger} a_{n+1}^{[2]} W_{0}^{-\dagger} & n=0,1 \ldots, \\ \frac{d}{d t}\left(W_{0}^{\dagger} a_{n+1}^{[2]} W_{0}^{-\dagger}\right)=W_{0}^{\dagger} a_{n+1}^{[2]} b_{n}^{[2]} W_{0}^{-\dagger}-W_{0}^{\dagger} b_{n+1}^{[2]} a_{n+1}^{[2]} W_{0}^{-\dagger}, & n=0,1, \ldots\end{cases}
$$

Proof. First of all observe that if we take derivative to $H_{n}=V_{n} W_{n}^{-1}$ then, we obtain the result given in Lemma 2. From the fact that $\frac{d}{d t}\left(H_{n} W_{0}\right)=-b_{n} H_{n} W_{0}$ and taking into account that $b_{n}^{[2] \dagger}=H_{n}^{-1} b_{n} H_{n}$ and $a_{n}^{[2] \dagger}=H_{n-1}^{-1} H_{n}$ we get the desired result.

Proposition 10. Let $J_{1}$ be a solution of a Toda lattice as in (17) and $\left(P_{n}^{[1]}\right)_{n \in \mathbb{N}}$ its first family of matrix biorthogonal polynomials associated. Then, for every $n \in \mathbb{N}$,

$$
\dot{P}_{n}^{[1]}(x, t)=a_{n}(t) P_{n-1}^{[1]}(x, t) .
$$

Proof. From the relation $x P^{[1]}=J_{1} P^{[1]}$ (recall that we are taking the derivate on the variable $t$ ), we have

$$
x \dot{P}^{[1]}=\dot{J}_{1} P^{[1]}+J_{1} \dot{P}^{[1]}=\left(J_{1-} J_{1}-J_{1} J_{1-}\right) P^{[1]}+J_{1} \dot{P}^{[1]} .
$$

From here

$$
\left(x I-J_{1}\right)\left(\dot{P}^{[1]}-J_{1-} P^{[1]}\right)=\mathbf{0} .
$$

So we get the result.

Proposition 11. If $J_{1}$ is a solution of a Toda lattice, then for all $n=1,2 \ldots$ the following identity is satisfied

$$
\left(\dot{J}_{1}^{n}\right)=J_{1-} J_{1}^{n}-J_{1}^{n} J_{1-} .
$$

Proof. The proof will be by induction. For $n=1$, it follows from (19). For $n=2$ we have

$$
\begin{aligned}
\dot{J}_{1}^{2} & =\dot{J}_{1} J_{1}+J_{1} \dot{J}_{1}=\left(J_{1-} J_{1}-J_{1} J_{1-}\right) J_{1}+J_{1}\left(J_{1-} J_{1}-J_{1} J_{1-}\right) \\
& =J_{1-} J_{1}^{2}-J_{1}^{2} J_{1-} .
\end{aligned}
$$


Suppose that the property is satisfied by $n=m-1$ to prove by $n=m$

$$
\begin{aligned}
\dot{J}_{1}^{m} & =\dot{J}_{1}^{m-1} J_{1}+J_{1}^{m-1} \dot{J}_{1} \\
& =\left(J_{1-} J_{1}^{m-1}-J_{1}^{m-1} J_{1-}\right) J_{1}+J_{1}^{m-1}\left(J_{1-} J_{1}-J_{1} J_{1-}\right) \\
& =J_{1-} J_{1}^{m}-J_{1}^{m} J_{1-} .
\end{aligned}
$$

thus for every $n \in \mathbb{N}$ we get the result.

Corollary 1. Let $u$ be a matrix of linear functionals such that the corresponding block Jacobi matrix is a solution of (17). Then the sequence of matrix moments $\left(u_{n}\right)_{n \in \mathbb{N}}$ satisfies the following matrix differential equation

$$
\dot{u}_{n}(t)=u_{n}(t) u_{1}(t)-u_{n+1}(t), \quad n \in \mathbb{N} .
$$

Proof. The result follows from the fact that $u_{n}(t)=\left(J_{1}^{n}\right)_{0,0}$.

Corollary 2. Let $u$ be a matrix of linear functionals such that its corresponding block Jacobi matrix is a solution of (17). Then its associated Stieltjes function satisfies the following matrix differential equation

$$
\dot{F}(z)=F(z)\left(u_{1}(t)-z \mathrm{I}\right)+\mathrm{I} .
$$

Proof. Using Corollary 1 we have that

$$
\dot{F}(z)=\sum_{k=0}^{\infty} \frac{\dot{u}_{k}(t)}{z^{k+1}}=F(z) u_{1}(t)-z \sum_{k=0}^{\infty} \frac{u_{n+1}(t)}{z^{n+2}},
$$

and the result follows from the definition of $F(z)$.

Proposition 12. If $J_{1}$ is a solution of a Toda lattice as in (17) and $u$ is the corresponding matrix of linear functionals, then the following equation holds

$$
\dot{u}(t)=u(t) u_{1}(t)-x u(t) .
$$

Proof. Using the original representation of the Stieltjes matrix function $F(z)$ and $(22)$ we have

$$
\begin{aligned}
\dot{F}(z) & =\left\langle\frac{\mathrm{I}}{z-x}, u_{1}(t)^{\dagger}-\bar{z} \mathrm{I}\right\rangle_{u(t)}+\langle\mathrm{I}, \mathrm{I}\rangle_{u(t)}, \\
& =\left\langle\frac{\mathrm{I}}{z-x}, u_{1}(t)^{\dagger}\right\rangle_{u(t)}+\left\langle\frac{z}{z-x}, \mathrm{I}\right\rangle_{u(t)}+\langle\mathrm{I}, \mathrm{I}\rangle_{u(t)},
\end{aligned}
$$

and so

$$
\dot{F}(z)=\left\langle\frac{\mathrm{I}}{z-x}, \mathrm{I}\right\rangle_{u(t) u_{1}(t)}-\left\langle\frac{\mathrm{I}}{z-x}, \mathrm{I}\right\rangle_{x u(t)} .
$$


Thus, if we denote by $\hat{u}$ the matrix of linear functionals

$$
\hat{u}=-\frac{d}{d t} u+u u_{1}(t)-u x,
$$

then the corresponding Stieltjes function is the zero matrix; but this implies that every matrix moment of $\hat{u}$ is equal to $\mathbf{0}$, and from here $\hat{u}$ is equal to the zero matrix of linear functionals.

Corollary 3. If in particular $\langle P, Q\rangle_{u}=\int P W(x, t) Q^{\dagger} d x$ and its correspondingJacobi matrix $J_{1}$ satisfies (17), then the matrix weight function $W(x, t)$ has the structure

$$
W(x, t)=e^{-x t} W(x, 0) K(t),
$$

where $K(t)$ satisfies the following matrix differential equation

$$
\dot{K}(t)=K(t) u_{1}(t) .
$$

Proof. Recall that if $J_{1}$ satisfies (17), then $u$ satisfies (23), but this implies that

$$
\dot{W}(x, t)=W(x, t)\left(u_{1}(t)-x \mathrm{I}\right) .
$$

On the other hand, if we take derivative in (24),

$$
\dot{W}(x, t)=W(x, t)\left(K^{-1}(t) \dot{K}(t)-x \mathrm{I}\right),
$$

and taking into account our hypothesis, we get the result.

Suppose now that we have a Jacobi matrix $J_{1}(t)$ with block LU factorization as in (10). If the sequence of matrix $\left(\gamma_{n}\right)_{n \in \mathbb{N}}$ is a solution of a Volterra lattice, i.e.

$$
\dot{\gamma}_{n+1}(t)=\gamma_{n+1}(t) \gamma_{n}(t)-\gamma_{n+2}(t) \gamma_{n+1}(t), \quad n \geq 0,
$$

then using the representation (13) it is easy to prove that $J_{1}$ and $\widetilde{J}_{1}$ are solutions of the Toda lattice (17). Here we are interested in the reciprocal result.

Theorem 1. If $\left(b_{n}\right)_{n \in \mathbb{N}}$ and $\left(a_{n}\right)_{n \in \mathbb{N}}$ are solutions of the Toda lattice (17) and their associate Jacobi block matrix $J_{1}$ has a block LU factorization, then there exists another Toda solution $\widetilde{J}_{1}$ and a Volterra solution such that (13) and (14) are verified.

Proof. First of all, notice that

$$
\frac{d}{d t}\left(P_{n}^{[1]}(x, t)^{-1}\right)=-P_{n}^{[1]}(x, t)^{-1}\left(\frac{d}{d t} P_{n}^{[1]}(x, t)\right) P_{n}^{[1]}(x, t)^{-1} .
$$

Let $\widetilde{J}_{1}$ be as in (12). If we take derivative in (16), we get that $\widetilde{J}_{1}$ is also a solution of the Toda lattice. Now, defining the sequence of matrices $\left(\gamma_{n}\right)_{n \in \mathbb{N}}$ as in (15), and taking 
derivative we obtain

$$
\begin{aligned}
& \dot{\gamma}_{2 n+1}=-a_{n+1} P_{n+1}^{[1]}(0, t)\left(P_{n}^{[1]}(0, t)\right)^{-1} \\
& \quad+P_{n+1}^{[1]}(0, t)\left(P_{n}^{[1]}(0, t)\right)^{-1} a_{n}(t) P_{n-1}^{[1]}(0, t)\left(P_{n}^{[1]}(0, t)\right)^{-1} \\
&=-\gamma_{2 n+2} \gamma_{2 n+1}+\gamma_{2 n+1} \gamma_{2 n}, \\
& \dot{\gamma}_{2 n}=-\left(a_{n} b_{n-1}-b_{n} a_{n}\right) P_{n-1}^{[1]}(0, t)\left(P_{n}^{[1]}(0, t)\right)^{-1} \\
& \quad-a_{n} a_{n-1} P_{n-2}^{[1]}(0, t)\left(P_{n}^{[1]}(0, t)\right)^{-1}-\left(a_{n} P_{n-1}^{[1]}(0, t)\left(P_{n}^{[1]}(0, t)\right)^{-1}\right)^{2} ;
\end{aligned}
$$

now, as $b_{n}=-P_{n+1}^{[1]}(0, t)\left(P_{n}^{[1]}(0, t)\right)^{-1}-a_{n} P_{n-1}^{[1]}(0, t)\left(P_{n}^{[1]}(0, t)\right)^{-1}$ we get that

$$
\begin{aligned}
\dot{\gamma}_{2 n} & =a_{n}(t)-P_{n+1}^{[1]}(0, t)\left(P_{n}^{[1]}(0, t)\right)^{-1} a_{n}(t) P_{n-1}^{[1]}(0, t)\left(P_{n}^{[1]}(0, t)\right)^{-1} \\
& =\gamma_{2 n} \gamma_{2 n-1}-\gamma_{2 n+1} \gamma_{2 n},
\end{aligned}
$$

and the result follows.

Remark 2. Observe that from the above Theorem, if $\left(b_{n}\right)_{n \in \mathbb{N}}$ and $\left(a_{n}\right)_{n \in \mathbb{N}}$ are solutions of the Toda lattice (17) and we know the sequence of matrix polynomials $\left(P_{n}^{[1]}(x, t)\right)_{n \in \mathbb{N}}$, that satisfies the recurrence relation (18) and $P_{n}^{[1]}(0, t), n \geq 0$, is a nonsingular matrix, then the matrix sequences $\left(\widetilde{a}_{n}(x)\right)_{n \in \mathbb{N}}$ and $\left(\widetilde{b}_{n}(x)\right)_{n \in \mathbb{N}}$ defined by

$$
\begin{aligned}
& \widetilde{b}_{n}(t)=P_{n+2}^{[1]}(0, t)\left(P_{n+1}^{[1]}(0, t)\right)^{-1}+b_{n+1}(t)-P_{n+1}(0, t)\left(P_{n}^{[1]}(0, t)\right)^{-1}, \\
& \widetilde{a}_{n}(t)=P_{n+1}^{[1]}(0, t)\left(P_{n}^{[1]}(0, t)\right)^{-1} a_{n}(t) P_{n-1}^{[1]}(0, t)\left(P_{n}^{[1]}(0, t)\right)^{-1},
\end{aligned}
$$

are also solution of the matrix Toda lattice (17). Moreover, in this case

$$
\begin{aligned}
\gamma_{2 n+1}(t) & =-P_{n+1}^{[1]}(0, t)\left(P_{n}^{[1]}(0, t)\right)^{-1}, \\
\gamma_{2 n}(t) & =-a_{n}(t) P_{n-1}^{[i]}(0, t)\left(P_{n}^{[i]}(0, t)\right)^{-1},
\end{aligned}
$$

is solution of a matrix Volterra lattice.

Next, we are going to show an illustrative example.

Example 1. Suppose that we have the following matrix weight

$$
\left.W(x, t)=e^{(1-x) t}\left[\begin{array}{cc}
x & -1 \\
0 & x
\end{array}\right] e^{-x} x^{\alpha}, \quad x \in\right] 0, \infty[, \quad \alpha>-1 .
$$

Observe that in this case $\int W(x, t) d x$ is an invertible matrix different from the identity. However, this hypothesis is not necessary in the symmetrization process. If $\left(P_{n}^{[1]}\right)_{n \in \mathbb{N}}$ is the sequence of left matrix orthogonal polynomials with respect to $W(x, t)$, then making a suitable change of the variable, we obtain that

$$
P_{n}^{[1]}(x, t)=\frac{1}{(1+t)^{n}} Q_{n}^{[1]}((t+1) x),
$$


where

$$
Q_{n}^{[1]}(x)=\left[\begin{array}{cc}
1 & 1 \\
0 & \frac{1}{(t+1)}
\end{array}\right]\left[\begin{array}{cc}
L_{n}^{\alpha+1}(x) & -\frac{n}{\alpha+1} L_{n-1}^{\alpha+2}(x) \\
0 & L_{n}^{\alpha+1}(x)
\end{array}\right]\left[\begin{array}{cc}
1 & -(t+1) \\
0 & (t+1)
\end{array}\right],
$$

and $\left(L_{n}^{\alpha}\right)_{n \in \mathbb{N}}$ is the sequence of scalar monic Laguerre polynomials of parameter $\alpha$ which are orthogonal with respect to the measure $d \mu=: e^{-x} x^{\alpha} d x$ and has the following monomial representation (see [9] for other characterizations),

$$
L_{n}^{\alpha}(x)=\frac{(-1)^{n}}{n !} \sum_{k=0}^{n}\left(\begin{array}{l}
n+\alpha \\
n-k
\end{array}\right) \frac{(-x)^{k}}{k !} .
$$

On the other hand, we know that $P_{n}^{[1]}(x, t)$ satisfies a three term recurrence relation

$$
P_{n+1}^{[1]}(x, t)=\left(x \mathrm{I}-b_{n}(t)\right) P_{n}^{[1]}(x, t)-a_{n}(t) P_{n-1}^{[1]}(x, t) .
$$

Using the representation given in (26) and the properties of the Laguerre polynomials, we find that

$$
b_{n}(t)=\left[\begin{array}{cc}
\frac{2+\alpha+2 n}{t+1} & -\frac{2}{1+\alpha} \\
0 & \frac{2+\alpha+2 n}{t+1}
\end{array}\right], \quad a_{n}(t)=\left[\begin{array}{cc}
\frac{n(\alpha+n+1)}{(t+1)^{2}} & 0 \\
0 & \frac{n(\alpha+n+1)}{(t+1)^{2}}
\end{array}\right],
$$

and

$$
H_{n}=\frac{e^{t} n ! \Gamma(\alpha+n+2)}{(t+1)^{\alpha+2 n+2}}\left[\begin{array}{cc}
1 & -\frac{(t+1)}{\alpha+1} \\
0 & 1
\end{array}\right] .
$$

Besides, observe that $\left(b_{n}\right)_{n \in \mathbb{N}}$ and $\left(a_{n}\right)_{n \in \mathbb{N}}$ satisfy the Toda lattice (17). Moreover, from the representation (26) and 27)

$$
P_{n}^{[1]}(0)=\frac{1}{(t+1)^{n}}\left[\begin{array}{cc}
1 & 1 \\
0 & \frac{1}{(t+1)}
\end{array}\right]\left[\begin{array}{cc}
L_{n}^{\alpha+1}(0) & -\frac{n}{\alpha+1} L_{n-1}^{\alpha+2}(0) \\
0 & L_{n}^{\alpha+1}(0)
\end{array}\right]\left[\begin{array}{cc}
1 & -(t+1) \\
0 & (t+1)
\end{array}\right],
$$

where $L_{n}^{\alpha}(0)=(-1)^{n} \frac{\Gamma(\alpha+n+1)}{\Gamma(\alpha+1)}$. Thus, if we use the symmetrization process we obtain that

$$
\gamma_{2 n+1}(t)=\frac{\alpha+n+2}{t+1}\left[\begin{array}{cc}
1 & \frac{(t+1)}{(\alpha+1)(\alpha+2)} \\
0 & 1
\end{array}\right], \quad \gamma_{2 n}(t)=\frac{n}{t+1}\left[\begin{array}{cc}
1 & -\frac{(t+1)}{(\alpha+1)(\alpha+2)} \\
0 & 1
\end{array}\right] .
$$

It is not difficult to check that the sequence of matrices $\left(\gamma_{n}\right)_{n \in \mathbb{N}}$ satisfies 25] and consequently we can construct a second solution of the matrix Toda lattice. Moreover, defining the following sequence of matrix polynomials, $\left(S_{n}^{[1]}\right)_{n \in \mathbb{N}}$, by

$$
\begin{gathered}
S_{2 n}^{[1]}(x, t)=\left[\begin{array}{cc}
\frac{1}{(t+1)^{n}} & \frac{1}{(t+1)^{n}} \\
0 & \frac{1}{(t+1)^{n+1}}
\end{array}\right]\left[\begin{array}{cc}
L_{n}^{\alpha+1}\left((t+1) x^{2}\right) & -\frac{n}{\alpha+1} L_{n-1}^{\alpha+2}\left((t+1) x^{2}\right) \\
0 & L_{n}^{\alpha+1}\left((t+1) x^{2}\right)
\end{array}\right]\left[\begin{array}{cc}
1 & -(t+1) \\
0 & (t+1)
\end{array}\right], \\
S_{2 n+1}^{[1]}(x, t)=\left[\begin{array}{cc}
\frac{x}{(t+1)^{n}} & \frac{x}{(t+1)^{n}} \\
0 & \frac{x}{(t+1)^{n+1}}
\end{array}\right]\left[\begin{array}{cc}
L_{n}^{\alpha+2}\left((t+1) x^{2}\right) & -\frac{n}{\alpha+3} L_{n-1}^{\alpha+2}\left((t+1) x^{2}\right) \\
0 & L_{n}^{\alpha+2}\left((t+1) x^{2}\right)
\end{array}\right]\left[\begin{array}{cc}
1 & -(t+1) \\
0 & (t+1)
\end{array}\right],
\end{gathered}
$$


then, we have that

$$
x S_{n}^{[1]}(x, t)=S_{n+1}^{[1]}(x, t)+\gamma_{n}(t) S_{n-1}^{[1]}(x, t) .
$$

We can also see that $\left(S_{n}^{[1]}\right)_{n \in \mathbb{N}}$ is the first family of matrix biorthogonal polynomials with respect to the matrix weight

$$
W(x, t)=e^{\left(1-x^{2}\right) t \mathrm{I}}\left[\begin{array}{cc}
x^{2} & -1 \\
0 & x^{2}
\end{array}\right] e^{-x^{2}}|x|^{2 \alpha+1}, \quad x \in \mathbb{R} .
$$

Due to the close relation between the matrix lattices of Toda and Volterra we are going to study the properties of the last one.

\section{Matrix Volterra System}

Suppose that we have the following matrix Volterra system

$$
\dot{\gamma}_{n+1}(t)=\gamma_{n+1}(t) \gamma_{n}(t)-\gamma_{n+2}(t) \gamma_{n+1}(t), \quad n \geq 0 .
$$

This system can be described in terms of a Lax pair $\left(\Gamma_{1}, \Gamma_{1-}^{2}(t)\right)$, i.e.

$$
\dot{\Gamma}_{1}(t)=\Gamma_{1-}^{2} \Gamma_{1}-\Gamma_{1} \Gamma_{1-}^{2},
$$

where $\Gamma_{1}(t)$ and $\Gamma_{1-}^{2}(t)$ are the following block matrices

$$
\Gamma_{1}(t)=\left[\begin{array}{ccccc}
\mathbf{0} & \mathrm{I} & & & \\
\gamma_{1}(t) & \mathbf{0} & \mathrm{I} & & \\
\mathbf{0} & \gamma_{2}(t) & \mathbf{0} & \mathrm{I} & \\
& \mathbf{0} & \gamma_{2}(t) & \mathbf{0} & \ddots \\
& & \ddots & \ddots & \ddots
\end{array}\right] \quad \text { and } \quad \Gamma_{1-}^{2}(t)=\left[\begin{array}{ccc}
\mathbf{0} & \mathbf{0} & \cdots \\
\gamma_{2}(t) \gamma_{1}(t) & \mathbf{0} & \cdots \\
\mathbf{0} & \gamma_{3}(t) \gamma_{2}(t) & \\
\vdots & \vdots & \ddots
\end{array}\right] .
$$

Observe that for each $t \in \mathbb{R}$, we have associated to the matrix $\Gamma_{1}(t)$ a sequence of matrix polynomials $\left(S_{n}^{[1]}\right)_{n \in \mathbb{N}}$ defined by

$$
S_{n+1}^{[1]}(x, t)=x S_{n}^{[1]}(x, t)-\gamma_{n}(t) S_{n-1}^{[1]}(x, t),
$$

or, in more compact form,

$$
S^{[1]} \Gamma_{1}(t)=x S^{[1]}, \quad \text { where, } \quad S^{[1]}=\left[S_{0}^{[1] \dagger}(x, t), S_{1}^{[1] \dagger}(x, t) \cdots\right]^{\dagger} .
$$

We will denote by $\mathfrak{s}$ the matrix of linear functionals associated with $\left(S_{n}^{[1]}\right)_{n \in \mathbb{N}}$, and $\left(w_{n}\right)_{n \in \mathbb{N}}$ will be the corresponding sequence of moment matrices.

Theorem 2. The following conditions are equivalent

(a) $\left(\gamma_{n}\right)_{n \in \mathbb{N}}$ is a solution of (28), i.e. $\dot{\Gamma}_{1}(t)=\Gamma_{1-}^{2} \Gamma_{1}-\Gamma_{1} \Gamma_{1-}^{2}$.

(b) For $n \in \mathbb{N}$,

$$
\frac{d}{d t}\left(\Gamma_{1}^{n}\right)_{0,0}=\left(\Gamma_{1}^{2}\right)_{0,0}\left(\Gamma_{1}^{n}\right)_{0,0}+\left(\Gamma_{1}^{n}\right)_{0,2}\left(\Gamma_{1}^{2}\right)_{2,0} .
$$

(c) For $n \in \mathbb{N}$, the moments satisfy that $\dot{w}_{n}=w_{n} w_{2}-w_{n+2}$. 
(d) The Stieltjes function satisfies the following differential equation

$$
\dot{F}(z)=F(z)\left(w_{2}(t)-z^{2} \mathrm{I}\right)+z w_{0}(t) .
$$

(e) The following equations for the matrix of the moment functionals hold

$$
\dot{\mathfrak{s}}(t)=\mathfrak{s}(t) w_{2}(t)-x^{2} \mathfrak{s}(t) .
$$

(f) If $\left(S_{n}^{[1]}\right)_{n \in \mathbb{N}}$ is the first sequence of matrix orthogonal polynomials associated with $\Gamma_{1}$, then

$$
\frac{d}{d t} S_{n}^{[1]}(x, t)=\gamma_{n}(t) \gamma_{n-1}(t) S_{n-2}^{[1]}(x, t)
$$

Proof. We will prove this theorem according to the following scheme
(a) $\Rightarrow$
(b) $\Rightarrow$ (c) $\Rightarrow$
(d) $\Rightarrow$
(e) $\Rightarrow$
(f) $\Rightarrow$ (a) .

We begin proving that $(a) \Rightarrow(b)$. First of all we establish that (a) implies that

$$
\dot{\Gamma}_{1}^{n}=\Gamma_{1-}^{2} \Gamma_{1}^{n}-\Gamma_{1}^{n} \Gamma_{1-}^{2}, \quad n \in \mathbb{N}
$$

The proof will be by induction. For $n=2$ we have

$$
\begin{aligned}
\left(\dot{\Gamma}_{1}^{2}\right) & =\dot{\Gamma}_{1} \Gamma_{1}+\Gamma_{1} \dot{\Gamma}_{1}=\left(\Gamma_{1-}^{2} \Gamma_{1}-\Gamma_{1} \Gamma_{1-}^{2}\right) \Gamma_{1}+\Gamma_{1}\left(\Gamma_{1-}^{2} \Gamma_{1}-\Gamma_{1} \Gamma_{1-}^{2}\right) \\
& =\Gamma_{1-}^{2} \Gamma_{1}^{2}-\Gamma_{1}^{2} \Gamma_{1-}^{2} .
\end{aligned}
$$

Suppose that 35 is satisfied by $n=m-1$, to prove by $n=m$.

$$
\begin{aligned}
\left(\dot{\Gamma}_{1}^{m}\right) & =\left(\dot{\Gamma}_{1}^{m-1}\right) \Gamma_{1}+\Gamma_{1}^{m-1} \dot{\Gamma}_{1} \\
& =\left(\Gamma_{1-}^{2} \Gamma_{1}^{m-1}-\Gamma_{1}^{m-1} \Gamma_{1-}^{2}\right) \Gamma_{1}+\Gamma_{1}^{m-1}\left(\Gamma_{1-}^{2} \Gamma_{1}-\Gamma_{1} \Gamma_{1-}^{2}\right) \\
& =\Gamma_{1-}^{2} \Gamma_{1}^{m}-\Gamma_{1}^{m} \Gamma_{1-}^{2} .
\end{aligned}
$$

To prove (31), we note that

$$
\left(\dot{\Gamma}_{1}^{n}\right)_{0,0}=\sum_{k=0}^{\infty}\left(\Gamma_{1-}^{2}\right)_{0, k}\left(\Gamma_{1}^{n}\right)_{k, 0}-\left(\Gamma_{1}^{n}\right)_{0, k}\left(\Gamma_{1-}^{2}\right)_{k, 0}=-\left(\Gamma_{1}^{n}\right)_{0,2}\left(\Gamma_{1-}^{2}\right)_{2,0} .
$$

On the other hand, is not difficult to check that $\left(\Gamma_{1-}^{2}\right)_{2,0}=\left(\Gamma_{1}^{2}\right)_{2,0}$ and

$$
\left(\Gamma_{1}^{n+2}\right)_{0,0}=\left(\Gamma_{1}^{n}\right)_{0,0}\left(\Gamma_{1}^{2}\right)_{0,0}+\left(\Gamma_{1}^{n}\right)_{0,2}\left(\Gamma_{1}^{2}\right)_{2,0} .
$$

From here

$$
\begin{aligned}
\left(\dot{\Gamma}_{1}^{n}\right)_{0,0} & =-\left(\Gamma_{1}^{n}\right)_{0,2}\left(\Gamma_{1-}^{2}\right)_{2,0}-\left(\Gamma_{1}^{n}\right)_{0,0}\left(\Gamma_{1-}^{2}\right)_{0,0}+\left(\Gamma_{1}^{n}\right)_{0,0}\left(\Gamma_{1-}^{2}\right)_{0,0} \\
& =\left(\Gamma_{1}^{n}\right)_{0,0}\left(\Gamma_{1-}^{2}\right)_{0,0}-\left(\Gamma_{1}^{n+2}\right)_{0,0} .
\end{aligned}
$$

To prove that $(b) \Rightarrow(c)$, we use (31) and Proposition 5 to obtain

$$
\dot{w}_{n}=w_{n} w_{2}-w_{n+2} \text {. }
$$


To prove that $(c) \Rightarrow(d)$, we use the definition of the Stieltjes function, and the fact that $w_{1}(t)=0$.

$$
\begin{aligned}
\dot{F}(z) & =\sum_{k=0}^{\infty} \frac{\dot{w}_{k}(t)}{z^{k+1}}=\sum_{k=0}^{\infty} \frac{w_{k}(t)}{z^{k+1}} w_{2}(t)-\frac{w_{k+2}(t)}{z^{k+1}} \\
& =F(z)\left(w_{2}(t)-z^{2} \mathrm{I}\right)+z w_{0}(t) .
\end{aligned}
$$

To prove that $(c) \Rightarrow(d)$, we use the original representation of the Stieltjes function $F(z)$. Notice that from (32), we have that

$$
\begin{aligned}
\dot{F}(z) & =\left\langle\frac{\mathrm{I}}{z-x}, w_{2}(t)^{\dagger}-\bar{z}^{2} \mathrm{I}\right\rangle_{\mathfrak{s}(t)}+z\langle\mathrm{I}, \mathrm{I}\rangle_{\mathfrak{s}(t)}+\langle x \mathrm{I}, \mathrm{I}\rangle_{\mathfrak{s}(t)} \\
& =\left\langle\frac{\mathrm{I}}{z-x}, w_{2}(t)^{\dagger}-\bar{z}^{2} \mathrm{I}\right\rangle_{\mathfrak{s}(t)}+z\left\langle\frac{z-x}{z-x} \mathrm{I}, \mathrm{I}\right\rangle_{\mathfrak{s}(t)}+\left\langle x \frac{z-x}{z-x} \mathrm{I}, \mathrm{I}\right\rangle_{\mathfrak{s}(t)} \\
& =\left\langle\frac{\mathrm{I}}{z-x}, w_{2}(t)^{\dagger}\right\rangle_{\mathfrak{s}(t)}-\left\langle x^{2} \frac{\mathrm{I}}{z-x}, \mathrm{I}\right\rangle_{\mathfrak{s}(t)} \\
& =\left\langle\frac{\mathrm{I}}{z-x}, \mathrm{I}\right\rangle_{\mathfrak{s}(t) w_{2}(t)}-\left\langle\frac{\mathrm{I}}{z-x}, \mathrm{I}\right\rangle_{x^{2} \mathfrak{s}(t)} .
\end{aligned}
$$

Thus, if we denote by $\hat{\mathfrak{s}}$ the matrix of linear functionals $\hat{\mathfrak{s}}=\frac{d}{d t} \mathfrak{s}-\mathfrak{s}(t) w_{2}(t)-\mathfrak{s}(t) x^{2}$, then its corresponding Stieltjes function is equal to the zero matrix, but this implies that every moment matrix of $\hat{\mathfrak{s}}$ is equal to $\mathbf{0}$, and from here $\hat{\mathfrak{s}}$ is equal to the zero matrix of linear functionals.

To prove that $(d) \Rightarrow(e)$, we use the fact that $\dot{S}_{n}^{[1]}(x, t)-\gamma_{n} \gamma_{n-1} S_{n-2}^{[1]}(x, t)$ is a matrix polynomial of degree less or equal to $n-1$. Let $m=0, \ldots, n-1$. From the hypothesis

$$
\begin{aligned}
0_{N} & =\frac{d}{d t}\left\langle S_{n}^{[1]}(x, t), x^{m} \mathrm{I}\right\rangle_{\mathfrak{s}}=\left\langle S_{n}^{[1]}(x, t), x^{m} \mathrm{I}\right\rangle_{\dot{\mathfrak{s}}}+\left\langle\dot{S}_{n}^{[1]}(x, t), x^{m} \mathrm{I}\right\rangle_{\mathfrak{s}} \\
& =\left\langle S_{n}^{[1]}(x, t), x^{m} \mathrm{I}\right\rangle_{\mathfrak{s}} w_{2}(t)-\left\langle x^{2} S_{n}^{[1]}(x, t), x^{m} \mathrm{I}\right\rangle_{\mathfrak{s}}+\left\langle\dot{S}_{n}^{[1]}(x, t), x^{m} \mathrm{I}\right\rangle_{\mathfrak{s}} \\
& =\left\langle\dot{S}_{n}^{[1]}(x, t)-\gamma_{n} \gamma_{n-1} S_{n-2}^{[1]}(x, t), x^{m} \mathrm{I}\right\rangle_{\mathfrak{s}},
\end{aligned}
$$

and it implies that $\dot{S}_{n}(x, t)=\gamma_{n} \gamma_{n-1} S_{n-2}(x, t)$.

Before proving that $(e) \Rightarrow(a)$ we observe that using the notation in (30) we can rewrite 34 as $\frac{d}{d t} S^{[1]}=\Gamma_{1-}^{2}(t) S^{[1]}$. With this in mind and recall that $S^{[1]} \Gamma_{1}(t)=x S^{[1]}$ we get

$$
\dot{\Gamma}_{1}(t) S^{[1]}(x, t)+\Gamma_{1}(t) \dot{S}^{[1]}(x, t)=x \dot{S}^{[1]}(x, t) .
$$

Thus,

$$
\begin{aligned}
\dot{\Gamma}_{1}(t) S^{[1]}(x, t)+\left(\Gamma_{1}(t)-x I\right) \Gamma_{1-}^{2}(t) S^{[1]}(x, t) & =\mathbf{0}, \\
\left(\dot{\Gamma}_{1}(t)-\Gamma_{1-}^{2} \Gamma_{1}+\Gamma_{1} \Gamma_{1-}^{2}\right) S^{[1]}(x, t) & =\mathbf{0} .
\end{aligned}
$$


And since for each $t,\left(P_{n}(x, t)\right)_{n \in \mathbb{N}}$ is a basis of left module $\mathbb{C}^{N \times N}[x]$ we conclude that $\dot{\Gamma}_{1}(t)-\Gamma_{1}^{2} \Gamma_{1}+\Gamma_{1} \Gamma_{1-}^{2}=\mathbf{0}$.

Corollary 4. If in particular $\langle P, Q\rangle_{\mathfrak{s}}=\int P W(x, t) Q^{\dagger} d x$ and its associated Jacobi matrix $\Gamma_{1}$ satisfies (28), then the matrix weight function $W(x, t)$ has the structure

$$
W(x, t)=e^{-x^{2} t} W(x, 0) K(t),
$$

where $K(t)$ satisfies the following matrix differential equation

$$
\dot{K}(t)=K(t) w_{2}(t) .
$$

Proof. Recall that from Theorem 2, $\Gamma_{1}$ satisfies (33), but this implies that

$$
\dot{W}(x, t)=W(x, t)\left(w_{2}(t)-x^{2} \mathbf{I}\right) .
$$

On the other hand, if we take derivative in 36 ,

$$
\dot{W}(x, t)=W(x, t)\left(K^{-1}(t) \dot{K}(t)-x^{2} \mathrm{I}\right) .
$$

and taking into account our hypothesis, we get the result.

Corollary 5 (Lax-type Theorem). Let $S^{[1]}$ be the block column vector of matrix monic polynomials $S_{n}^{[1]}(x, t)$ and Let $\lambda(t)$ be a spectral point of the Jacobi matrix $\Gamma_{1}(t)$, i.e.

$$
\Gamma_{1} S^{[1]}(\lambda(t))=\lambda(t) S^{[1]}(\lambda(t)) ;
$$

if $\Gamma_{1}(t)$ satisfies 29, , then $\lambda(t)$ does not depend on $t$.

Proof. Taking derivative in 37

$$
\dot{\Gamma}_{1} S^{[1]}(\lambda(t))+\Gamma_{1} \dot{S}^{[1]}(\lambda(t))=\dot{\lambda}(t) S^{[1]}(\lambda(t))+\lambda(t) \dot{S}^{[1]}(\lambda(t)) .
$$

Taking into account (29) we get

$$
\left(\lambda(t) I-\Gamma_{1}\right)\left(\Gamma_{1-}^{2} S^{[1]}(\lambda)-\dot{S}^{[1]}(\lambda)\right)=\dot{\lambda}(t) S^{[1]}(\lambda(t)) .
$$

And again using that $\dot{S}^{[1]}(x, t)=\Gamma_{1-}^{2} S^{[1]}(x, t)$ we get $\dot{\lambda}(t) S^{[1]}(\lambda(t))=\mathbf{0}$ but this implies that $\dot{\lambda}(t)=\mathbf{0}$.

Remark 3. We emphasize that we do not have the reciprocal of the last result.

In fact, if the spectral points of the Jacobi matrix $\Gamma_{1}(t)$ do not depend on $t$, then there exists a semi-infinite block matrix $C=\left[c_{j, k}\right]_{j, k=0}^{\infty}$ such that $\dot{\Gamma}_{1}=C \Gamma_{1}-\Gamma_{1} C$, where $C$ has 
the shape

$$
\left[\begin{array}{ccccccc}
\mathbf{0} & \mathbf{0} & \mathbf{0} & \mathbf{0} & \mathbf{0} & \mathbf{0} & \ldots \\
\mathbf{0} & \mathbf{0} & \mathbf{0} & \mathbf{0} & \mathbf{0} & \mathbf{0} & \ldots \\
* & \mathbf{0} & \mathbf{0} & \mathbf{0} & \mathbf{0} & \mathbf{0} & \ldots \\
\mathbf{0} & * & \mathbf{0} & \mathbf{0} & \mathbf{0} & \mathbf{0} & \ldots \\
* & \mathbf{0} & * & \mathbf{0} & \mathbf{0} & \mathbf{0} & \ldots \\
\mathbf{0} & * & \mathbf{0} & * & \mathbf{0} & \mathbf{0} & \ldots \\
* & \mathbf{0} & * & \mathbf{0} & * & \mathbf{0} & \ldots \\
\vdots & \ddots & \ddots & \ddots & \ddots & \ddots & \ddots
\end{array}\right]
$$

The sequence $\left(\gamma_{n}\right)_{n \in \mathbb{N}}$ must satisfy that

$$
\dot{\gamma}_{n}=c_{n, n-2}-c_{n+1, n-1}, \quad n \in \mathbb{N}
$$

moreover, for every $n>2$ and $m=2,3, \ldots$

$$
\mathbf{0}=c_{n, n-2 m}+c_{n, n-2(m-1)} \gamma_{n-2(m-1)}-\gamma_{n} c_{n-1, n-(2 m-1)}-c_{n+1, n-(2 m-1)} .
$$

Observe then that in this case we cannot assure that $C$ is equal to $\Gamma_{-}^{2}$. This is due among other things to the fact that any matrix high-order Volterra lattice also satisfies the Corollary 5 (see [5]), where we said that $\Gamma_{1}$ is a solution of a matrix high-order Volterra lattice if it satisfies that

$$
\dot{\Gamma}_{1}(t)=\left(\Gamma_{1_{-}}\right)^{2 m} \Gamma_{1}-\Gamma_{1}\left(\Gamma_{1-}\right)^{2 m},
$$

for some $m \in \mathbb{N}$.

We remark that all the results given here can be generalized, using similar techniques, to study this type of high order Volterra systems.

\section{ACKNOWLEDGEMENTS}

We thank the valuable suggestions, comments and criticism by the referees. They significantly improved the contents and presentation of the manuscript.

A. Branquinho thanks financial support from the Centre for Mathematics of the University of Coimbra UID/MAT/00324/2019, funded by the Portuguese Government through FCT/MCTES and co-funded by the European Regional Development Fund through the Partnership Agreement PT2020.

A. Foulquié acknowledges CIDMA Center for Research and Development in Mathematics and Applications (University of Aveiro) and the Portuguese Foundation for Science and Technology (FCT) within project UID/MAT/04106/2019.

J.C. García-Ardila thanks financial support from the Spanish "Ministerio de Economía y Competitividad" research project MTM2015-65888-C4-2-P, Ortogonalidad, teoría de la aproximación y aplicaciones en física matemática. 


\section{REFERENCES}

[1] C. Álvarez-Fernández, G. Ariznabarreta, J. C. García-Ardila, M. Mañas, F. Marcellán, Christoffel transformations for matrix orthogonal polynomials in the real line and the non-Abelian 2D Toda lattice hierarchy, Internat. Math. Res. Notices, 5 (2017), 1285-1341.

[2] A. I. Aptekarev, A. Branquinho, Padé approximants and complex high-order Toda lattices, Journal of Computational and Applied Mathematics 155 (2), 231-237 (2003).

[3] A.I. Aptekarev, A. Branquinho, F. Marcellán, Toda-type differential equations for the recurrence coefficients of orthogonal polynomials and Freud transformation, J. Comput. Appl. Math. 78 (1), 139-160 (1997).

[4] A. Branquinho and A. Foulquié Moreno, A. Mendes Dynamics and interpretation of some integrable systems via matrix orthogonal polynomials, Integral Transforms Spec. Funct. 28 (1), 74-90 (2017).

[5] D. Barrios-Rolanía, A. Branquinho, Complex high-order Toda and Volterra lattices, J. Difference Equ. Appl. 15 (2), 197-213 (2009).

[6] D. Barrios Rolanía, J. R. Gascón Márquez, Spectrum and generation of solutions of the Toda lattice, Discrete Dyn. Nat. Soc. 2009, 12 pp.

[7] D. Barrios-Rolanía, R. Hernández-Heredero, On the relation between the complex Toda and Volterra lattices, arXiv:nlin/0610010v1 [nlin.SI]

[8] M. I. Bueno, F. Marcellán, Darboux transformation and perturbation of linear functionals, Linear Algebra Appl. 384 (2004), 215-242.

[9] T.S. Chihara, An introduction to orthogonal polynomials, Gordon and Breach Science Publishers, New York (1978).

[10] I. M. Gel'fand, G. E. Shilov, Generalized Functions. Volume I: Properties and Operations, Academic Press, New York, 1964.

[11] F. Gesztesy, H. Holden, B. Simon, Z. Zhao, On the Toda and Kac-van Moerbeke systems, Trans. Amer. Math. Soc. 339 (2) (1993) 849-868.

[12] M. Hamanaka, K. Toda, Towards noncommutative integrable systems, Phys. Lett. A 316, 77-83 (2003).

[13] B. A. Kupershmidt, KP or mKP. Non-commutative mathematics of Lagrangian, Hamiltonian, and integrable systems, Providence: AMS, 2000.

[14] C. X. Li, J. J. C. Nimmo, Quasideterminant solutions of a non-Abelian Toda lattice and kink solutions of a matrix sine-Gordon equation, Proc. R. Soc. A 464, 951-966 (2008).

[15] A. V. Mikhailov, Integrability of the two-dimensional generalization of Toda chain, JETP Lett. 30, 443-447 (1979).

[16] J. J. C. Nimmo, R. Willox, Darboux transformations for the two-dimensional Toda system, Proc. R. Soc. A 453, 2497-2525 (1997).

[17] E. M. Nikishin, V. N. Sorokin, Rational approximations and orthogonality, Translated from the Russian by Ralph P. Boas, Translations of Mathematical Monographs, 92, Amer. Math. Soc., Providence, RI, 1991.

[18] L. D. Paniak, Exact noncommutative KP and KdV multi-solitons. arXiv:hep-th/0105185v2 (2001).

[19] F. Peherstorfer, On Toda lattices and orthogonal polynomials, J. Comput. Appl. Math. 133 (2001) 519-534.

[20] V. Spiridonov, A. Zhedanov, Discrete Darboux transformatios, the discrete-time Toda lattice, and the AskeyWilson polynomials, Meth. appl. Anal. 2 (1995) 283-305.

[21] V. Spiridonov, A. Zhedanov, Discrete-time Volterra chain and classical orthogonal polynomials, J. Phys. A: Math Gen. 30 (1997) 8727-8737. 